\title{
A Review on the Current Therapies for Schizophrenia
}

\author{
Javeria Sahib Din ${ }^{1}$, Ali Mahmood Khan ${ }^{2 *}$ and Usman Iqbal Jangda ${ }^{3}$ \\ ${ }^{1}$ Bergen Regional Medical Center, USA \\ ${ }^{2}$ Manhattan Psychiatric Center, USA \\ ${ }^{3}$ Zucker Hillside Hospital, USA
}

Submission: August 02, 2017; Published: August 07, 2017

*Corresponding author: Ali Mahmood Khan, Manhattan Psychiatric Center, USA; Email: ali_mahmood_khan@hotmail.com

\begin{abstract}
According to statistics, approximately 40 to 60 percent of Schizophrenics experience a life-long deterioration. In this article, we have presented an updated review of the current pharmacologic and non-pharmacologic treatment options of Schizophrenia. Anti-psychotics are the mainstay of treatment for Schizophrenia. CATIE study concluded that first generation anti-psychotics have similar efficacy as of second generation agents Manschreck [1]. Olanzapine had better results than other atypical anti-psychotics and ziprasidone was beneficial to avoid weight gain secondary to atypical anti-psychotic agents. In another meta-analysis, ziprasidone and aripiprazole have proved efficacy in maintaining BMI. Despite olanzapine's effect on BMI, second generation agents provide a better quality of life (QOL) than first generation antipsychotics Gründer [2]. In early phase of symptomatic disease, olanzapine and risperidone have proven beneficial in eradication of symptoms Furukawa [3]. In another trial, injectable form of risperidone was more effective in preventing relapse and in maintaining adherence to treatment regimen than the oral risperidone Subotnik [4]. Clozapine is the best option available for treatment resistant Schizophrenia. In ACLAIMS, a double blind clinical trial, Haldol decanoate and paliperidone palmitate were compared for efficacy and no significant difference in efficacy was found between the two forms.
\end{abstract}

In addition to appropriate pharmacotherapy, psychotherapeutic interventions like cognitive behavioral therapy, motivational interviewing, family intervention programs and coping mechanisms are discussed in detail in the article with respect to their proven efficacy by latest trials conducted Subotnik [4].

Abbreviations: QOL: Quality of Life; PANSS: Positive and negative syndrome scale; DPN: Diphenonucleotide; CATIE: Clinical Antipsychotic Trials of Intervention Effectiveness; FGA: First Generation Anti-psychotics; NMDAR: N-Methyl D-Aspartate Receptor; SGA: Second Generation Anti-psychotics; ACLAIMS: A Comparison of Long-acting Injectable Medications for Schizophrenia; NIMH: National Institute of Mental Health;

\section{Introduction}

It is interesting how human thought process works and how a slight incongruity in neurotransmitters can make the brain "go nuts". According to DSM-5, two of any of the five symptoms of delusions, any type of hallucinations, disorganized speech, disorganized behavior or abnormal behavior and negative symptoms must persist for at least six months to diagnose a person with Schizophrenia. Symptoms must be enough to cause functional decline. History of Schizophrenia dates back in 1887, when Kraepelin first recognized it as a distinct mental disorder. But the word "Schizophrenia" came less than hundred years ago. In the past, research scientists and physicians have strived to fight this debilitating illness by different means. Treatment of Schizophrenia has evolved over many years and now, a menu of options is available to fight this disease.
Schizophrenia is incapacitating not only for the person suffering from it, but, it exhausts the whole family and the society in general. According to a survey in 2002, the overall expenditure of Schizophrenia in USA is appraised to be 62.7 billion dollars yearly, among which 22.7 billion is spent explicitly on "healthcare". This 22.7 billion is a combination of 8 billion disbursed in long term care, 7 billion spent on outpatient, 5 billion on drugs and 2.8 billion expended in inpatient. This explains Crown et al.'s evaluation that approximately 40 to 60 percent of persons suffering from Schizophrenia will experience lifelong deterioration. The burden of Schizophrenia on the society involves not only the cost of healthcare but also unemployment, the strain on families involved in care, cost of law enforcement and shelter homes $\mathrm{Wu}$ [5]. 


\section{Current Treatments}

The aim of treating Schizophrenia is to conform to three goals, which include the eradication of symptoms, ensuring a higher quality of life and establishing compliance. In each patient encounter, the first step towards treatment involves reaching a correct diagnosis, with the help of clinical, psychosocial, laboratory and radiology outcomes. The approach to a correct "treatment modality" comes afterwards. This also involves the correct setting in which the Schizophrenia person will be getting services. Establishing a physician patient relationship is significant in the future compliance to treatment in every psychiatric patient, including the Schizophrenics. A 30-item Positive and negative syndrome scale (PANSS) was established to assess the relationship between positive and negative symptoms of Schizophrenia and the severity of illness. In this study on 101 Schizophrenics, it was pioneered that positive and negative scores were inversely correlated and hence "mutually exclusive" Kay [5].

\section{Medication Options}

Research scientists have endeavored for decades to find the best possible treatment for Schizophrenia. In 1966, diphenonucleotide (DPN) has been tested Bowers [6]. In 1982, a trial on Apo-morphine was conducted in the treatment of Schizophrenia Levy [7]. Unfortunately, it was effective in neither the treatment of psychosis nor tardive dyskinesia. Currently, there are pharmacologic and non-pharmacologic therapies available for rehabilitation of a Schizophrenia person. Despite a menu of options available in treating schizophrenia, anti-psychotics are considered the backbone of treatment for Schizophrenia, proven effective in hundreds of clinical trials. On the basis of data from 1995 to 2002, the pharmacologic options in the palliation of negative symptoms in a Schizophrenia person include typical and atypical antipsychotics, antidepressants, glutaminergic medications, estrogens and the anti-seizure medications Möller [8]. However, the addition of anti-epileptic drug to treatment regimen of negative symptoms is not fruitful according to available data.

In the past decade, head-to-head clinical trials have been conducted on the efficacy of second generation anti-psychotics over first generation anti-psychotics. Typical ant-psychotics i.e., first generation anti-psychotics, first came into the market in 1950s and Atypical anti-psychotics i.e., first became available in 1990s Clinical Antipsychotic Trials of Intervention Effectiveness (CATIE) was conducted to compare the first generation perphenazine with second generation agents. It was concluded after this trial that perphenazine has efficacy similar to second generation agents and does not show EPS in moderate doses. Olanzapine was proven to be beneficial than other atypical antipsychotics in this study despite its effects on weight gain and Diabetes Mellitus. Ziprasidone was effective in weight loss and clozapine for patients who had discontinued their medication in the phase 1 of the trial Manschreck (2007).
The first and second generation anti-psychotics for the treatment of Schizophrenia were compared in a meta-analysis Leucht [9]. Four of the second-generation anti-psychotics i.e., amisulpride, clozapine, olanzapine and risperidone exhibited better efficacy. Except these four second generation antipsychotics, none of the other medications of this class showed more efficacy than the first-generation anti-psychotics in this meta-analysis. Additionally, second generation anti-psychotics have lesser extra pyramidal side effects than haloperidol and some ofsecond generation have less EPS than low potency first generation anti-psychotics (FGA) Leucht [9]. Second generation anti-psychotics (SGA), except ziprasidone and aripiprazole; induce more weight gain than first generation anti-psychotics Leucht [9].

In another study, the Quality of Life (QOL) of patients receiving FGA and SGA was compared. Although, olanzapine causes weight gain and an increase in BMI, but those Schizophrenia persons receiving SGA had better QOL than those patients receiving FGA Gründer [2].

In the early severe phase of Schizophrenia, olanzapine and risperidone have ascertained to be valuable in elimination of symptoms. However, in mild symptoms of Schizophrenia, they did not show greater efficacy. The adverse events reported by the patient should always be weighed in comparison to the benefits of the anti-psychotic prescribed Furukawa [3]. Similarly, according to a randomized clinical trial in 2015 , in the early phase of Schizophrenia i.e., after the first episode of Schizophrenia, oral and injectable forms of second generation anti-psychotics were compared for their efficacy. Injectable forms of anti-psychotics are usually not prescribed after the first episode. This clinical trial served as a "game changer" and it demonstrated that long acting injectable risperidone surpassed correspondence to the medication regimen, prevention of relapse and dominated in the control of psychosis as compared to the oral formulation of risperidone. Additionally, injectable risperidone given after the first episode of Schizophrenia can also improve cognition Subotnik [4].

A considerable research has been executed about the add-on therapies of Schizophrenia. Previously, dopaminergic antagonism has been extensively studied as a countermeasure approach to anti-psychotics. A recently studied approach is the agonism of N-Methyl D-Aspartate Receptor (NMDAR), as a counteraction of hypoactive NMDAR, noticed in Schizophrenia Lane [10]. A double-blind, randomized control trial on the efficacy of Benzoic acid as an add-on therapy for Schizophrenia was performed. Benzoate's adequacy was proved in all areas of treatments in Schizophrenia such as cognition and positive symptoms. Neurocognition was assessed by scales recommended by National Institute of Mental Health (NIMH) i.e., PANSS total score, Scales for assessment of Negative Symptoms-20 items, Quality of Life scale (QOL), Global assessment of function, Clinical Global Impression and Improvement scale. Prior to 
this research, there has been debate on dietary Benzoic acid in terms of improving Schizophrenia symptoms Lane [10]. Similarly, in another trial D-Serine, which is a natural NMDAR modulator, has proven beneficial in the eradication of prodromal symptoms including the negative symptoms in Schizophrenics. D-Serine was specifically studied for the prodromal stage, as NMDAR agonists have been studied for full blown symptoms of Schizophrenia before Kantrowitz [11].

In a latest meta-analysis by Correll et al. [12], pharmacologic co-treatments in form of addition of another psychotropic medication to the anti-psychotic monotherapies for treatment of Schizophrenia were studied. The aim was to analyze the augmentation of overall symptom domain of Schizophrenia in relation to the anti-psychotic monotherapy. It was concluded in 21 interventions of the meta-analysis that cotreatments are beneficial. However, higher quality clinical trials are required for any further recommendation strategies regarding this idea Correll et al. [12].

Which Medications have proven Effective in Preventing Relapse of Schizophrenia in Clinical Trials?

"Relapse" is a term used in Schizophrenia when a patient fails his therapy, is re-hospitalized, is suicidal, declines to comply or dies. Clozapine, a second-generation anti-psychotic, has shown efficacy in prevention of these events. Similarly, long acting injections of anti-psychotics have proven beneficial in preventing relapse. However, oral formulations have elevated hazards of backsliding. This development is corresponding to the latest cohort study on 29,823 schizophrenic patient populations, published in July 2017 Tiihonen [13].

Now raises the question of which long acting injectable form to be used for maintenance of Schizophrenia. Should it be the first generation or the second-generation anti-psychotic? To answer this question, we have looked into the recent clinical trials in this regard. Haloperidol Decanoate is the long acting injectable form of first generation anti-psychotics, which has been traditionally used for a long time as a maintenance therapy of Schizophrenia. Paliperidone palmitate is a newer long acting injectable anti-psychotic, which is an active metabolite of Risperidone. Risperidone injectable form has been available since 2003 in the market. However, there were a few backsides to it i.e., it had to be administered every two weeks and required to be refrigerated. Whereas, in 2009, its active metabolite Paliperidone was introduced, this does not require refrigeration and is administered every month Mc Evoy [14].

ACLAIMS (A Comparison of Long-acting Injectable Medications for Schizophrenia) is a double blind clinical trial comparing Haloperidol Decanoate with Paliperidone Palmitate. No statistically significant difference was found between the efficacies of these two injectable forms. Similar conclusions were drawn from the previous CATIE trial, as discussed in the previous section of this article McEvoy [14].

\section{Which medications is Effective in Treatment Resistant Schizophrenia?}

Schizophrenia persons who do not recover cognitively, socially and psychologically and are unable to get back to a social setting are termed as treatment resistant. In the 1950s, when phenothiazines were introduced in the market, those patients which did not respond to the drug were considered treatment resistant. However, over the years the definition has changed. It is estimated that around $30 \%$ of Schizophrenia persons will show treatment resistance Mouchlianitis [15].

Presently, there is not enough data in form of blinded studies, to ascertain which drug is efficacious in this patient population. However, clozapine has noteworthy effects demonstrated consistently by meta-analysis Samara (2016) .Thus, clozapine is so far considered the drug if choice in treatment resistant Schizophrenia. In a systematic search on PUBMED from January 1980 to April 2015, neurologic changes on imaging of brain were studied. The reason of this search was to catalog neuroimaging changes and identify the patients at an earlier stage. Treatment resistant patients were consistently found to have reduced gray matters and perfused frontotemporal regions whereas treatment responsive group demonstrated increased white matter and perfusion of basal ganglia. Those patients treated with clozapine showed reduced caudate nucleus volume. Hence, there were some neurobiological changes found in treatment resistant group, which should be further studied in future Mouchlianitis [15].

Treatment Recommendations For Smoking and Substance use Disorder in Schizophrenia

The association of cigarette smoking as a risk factor for Schizophrenia is established and is currently being studied. However, the use of cannabis as a causal risk factor for Schizophrenia is also a question to many authors as cannabis use has a complicated association with smoking Gage [16]. In a meta-analysis, the association of daily tobacco smoking with earlier age of onset of and a higher risk of a psychotic illness have been studied by Gurillo and colleagues. Authors have also studied the relation between smoking and mortality. Smoking and other environmental risk factors can be a cause of increased mortality in Schizophrenia patients, and reducing these factors can decrease the mortality in Schizophrenia Gurillo [17]. Also, the use of tobacco is associated with poor outcomes of substance abuse treatment. Tobacco use minimizes the response to medications and increases the risk of suicidal behaviors. Hence, mental health professionals should treat tobacco use and substance use disorder along with the treatment regimen of Schizophrenia Prochaska [18]. 
Verinicline is an agonist at nicotinic acetylcholine receptor and prevents the binding of nicotine at $\alpha 4 \beta 2$ receptors. Hence, has gained popularity for the treatment of smoking cessation. The use of verinicline in smoking cessation for Schizophrenics is being extensively studied. In a double blind, placebo controlled trial; moderate dose of verinicline has beneficial effects in treating Schizophrenics with specific biomarkers. It had positive effects on spatial working memory, sustained attention and improved executive function and processing speed Hong [19].

\section{Effects of Different Therapies on Cognitive Impairment}

Cognitive impairment in Schizophrenia has biologic and genetic linkages, which can be beneficial when treating it Dickinson [20] In a recent blinded trial based on the results of 12 studies, the factors that effected cognition were studied by the standard Measurement and Treatment Research to Improve Cognition in Schizophrenia Consensus Cognitive Battery (MCCB). There were no significant differences found in the MCCB in treatment group and placebo groups Keefe [21].

\section{Psychotherapeutic Interventions}

According to APA guidelines, pharmacologic treatment is not the only intervention in patients with Schizophrenia, psychotherapeutic interventions are equally important. Medications reduce the psychotic symptoms but do not provide complete cure. Scaling down of symptoms does not necessarily mean one can function better socially. Psychotherapy and social support improve the functioning of a Schizophrenia person. These techniques can be used in acute and post-acute phases of Schizophrenia Pekkala et al. [22]. Describes in his Cochrane database the results of psycho education in Schizophrenia. According to his analysis, any type of psychotherapeutic intervention decreases the rate of relapse and readmission in Schizophrenia persons. This study showed positive effects of psychotherapies on the health of individuals with Schizophrenia Pekkala et al. [22].

\section{Cognitive Behavioral Therapy}

It has been estimated that around $75 \%$ of the Schizophrenics cease taking the medications 18 months after use due to changing or not taking the treatment regimen. This population has more frustration owing to treatment. Even in those compliant to their medications, $50 \%$ continue to experience the psychotic symptoms. Such cases can be avoided by timely psychotherapeutic intervention. Several trials and studies have demonstrated the efficaciousness of CBT in stabilization of psychosis related to Schizophrenia. Previously, CBT has been used to treat depression and anxiety, but now it has gained popularity in treating psychotic symptoms. It is used in adjunct to the pharmacotherapy for treating Schizophrenia. Cognitive behavioral therapy is a short-term treatment approach. Its objective is to modify the way patients think and hence, behave.
It inculcates positive attitudes towards daily life issues and emotional problems the patients face. CBT is usually given weekly and is goal-directed. The therapist and the physician are engaged in conversation for about 50 minutes. Patients are taught the principles of coping mechanisms which allow them to better function independently Rathod [23].

In a recent study in which the cost-effectiveness of different treatment regimens adopted for Schizophrenia was studied, it was demonstrated that the most cost-effectiveapproach was atypical anti-psychotics with psychotherapy Rathod [23]; Wykes et al. [24]. Explains in his meta-analysis the different effect sizes of various symptoms of Schizophrenia when targeted by CBT Wykes et al. [24] 2008). Positive effects on positive symptoms, negative symptoms, mood, affect and anxiety were noted but no effects on hopelessness were exhibited. In another "randomized control study for persistent symptoms of schizophrenia resistant to medications", CBT showed positive results in both the eradication of positive and negative symptoms when patients are followed for 9 months. These results are seen in Schizophrenics who are resistant to the effects of anti-psychotic medications Sensky [25].

\section{Family Intervention Programs}

Family intervention programs target the struggling Schizophrenic patients and their families. Relatives of Schizophrenics are professed on their behaviors with the patient. Also, it has been previously demonstrated that those patients whose families condemn and find faults in them, have a higher rate of relapsing and failing treatment Pharoah [26]. In a meta-analysis of 53 RCTs, it was demonstrated that family intervention might reduce the rate of relapse and hospital admissions. Additionally, they increase the compliance to treatment regimen in Schizophrenics Pharoah [26]. In another study, family intervention was studied by measuring the rate of relapse and readmission within one year of treatment. The results exhibited $20 \%$ reduction in the relapse of Schizophrenics whose families were also included in the treatment. It was also exhibited that aftereffects were pronounced if the therapy to the relatives was provided for longer than three months PitschelWalz [27].

\section{Coping Mechanisms}

Different persons react differently to the stressful situations. The procurement of skills to deal with the disability in Schizophrenia has several aspects. It includes denial and acceptance, knowledge of the illness, knowledge of the medications and drugs, delusional thinking, social communication skills, hallucinations or self-talking, emotions, stress, hobbies, stigma, networking and consumer groups. It is hard for a mentally ill person to believe that he is ill; such persons are approached by different ways to make them believe that others view you as mentally ill; hence they try to learn 
more about their illness. Paleologic Arieti, Brody and par-ataxic distortion Sullivan [28] is a part of Schizophrenia illness, which develops as a result of emotional stress the mentally ill persons undergo. Teaching them about their illness is another coping mechanism. It is estimated that $50 \%$ of Schizophrenics also have substance use disorder (SUD) in their lifetime. Nicotine and cannabis are the most frequently used. It is vital to teach them the association of comorbidity with the illness. Also, the medication that they receive, they should have knowledge of the importance of its compliance and effects on their health in long term Volkow [29].

ASchizophrenia person might misinterpret the conversations in the social settings and be at risk of losing jobs and friends. They should be given an insight into their social dysfunction by the therapist. In addition, it has been found that those places or situations where there is more "expressed emotion", it is harder for Schizophrenics to recover. They are advised to avoid such places and their relatives and close ones are taught not to criticize them. Not only emotions, Schizophrenics can also break down in stress and excitement. Organizations like National Alliance of Mentally Ill (NAMI), which was built in 1977, help the mentally ill and their families by providing social support and group therapies. They help them deal with the stigma of mental illness Carr [30].

\section{Motivational Interviewing (MI)}

Schizophrenia decreases the motivation in individuals momentously which in turn reduces the functionality of individuals in psychosocial settings. This cognitive decline leads to treatment non-adherence. Treatment non-adherence is one of the common reasons why Schizophrenics fail therapy. The psychotherapeutic approach which moves one from undetermined state towards a motivated state is termed as "motivational interviewing". Motivational interviewing based Adherence therapy is a technique which can cut back the severity of symptoms and the rate of re-hospitalization. It also improves the compliance to the treatment regimen in patients. Motivational interviewing also helps the patients to increase insight into the disease process. This approach is being adapted to increase adherence to treatment in psychosis as well. However, booster sessions of MI are always required for enhanced effects Fiszdon [31].

\section{Electroconvulsive Therapy}

Electroconvulsive therapy (ECT) is performed under general anesthesia. Controlled electric currents are passed through the brain to alter the neurotransmitters and the neurons in the brain. ECT was first used in 1938. Its use was markedly reduced after the introduction of antipsychotics and now it is used in certain severe treatment resistant Schizophrenics only. It is also helpful in catatonia of Schizophrenia, according to few authors Phutane [32]. According to a study, ECT was mostly given for patients who had less therapeutic response to the pharmacotherapy, those patients in whom urgent response to treatment was needed, and in those who have failed other treatments. The symptoms to which ECT most responds are catatonia, aggressive behavior and suicide. However, the symptom most responsive to ECT is catatonia Phutane [32,33].

\section{Conclusion}

Schizophrenia is a debilitating illness causing a huge economic burden on the healthcare system every year. Head to head clinical trials have been conducted to fight this mental illness. Appropriate pharmacotherapy and augmentation with psychotherapeutic techniques remains the mainstay of treatment. Anti-psychotics are the most widely used medications as a first line treatment.

\section{References}

1. Manschreck TC, Boshes RA (2007) The CATIE schizophrenia trial: results, impact, controversy. Harvard review of psychiatry Harv Rev Psychiatry 15(5): 245-258.

2. Gründer G, Heinze M, Cordes J, Mühlbauer B, Juckel G, et al. (2016) Effects of first-generation antipsychotics versus second-generation antipsychotics on quality of life in schizophrenia: a double-blind, randomised study. The Lancet Psychiatry 3(8): 717-729.

3. Furukawa TA, Levine SZ, Tanaka S, Goldberg Y, Samara M, et al (2015) Initial severity of schizophrenia and efficacy of antipsychotics: participant-level meta-analysis of 6 placebo-controlled studies. JAMA psychiatry: 72(1): 14-21.

4. Subotnik KL, Casaus LR, Ventura J, Luo JS, Hellemann GS, et al. (2015) Long-acting injectable risperidone for relapse prevention and control of breakthrough symptoms after a recent first episode of schizophrenia: a randomized clinical trial. JAMA psychiatry 72(8): 822-829.

5. Wu EQ, Birnbaum HG, Shi L, Ball DE, Kessle RC, et al. (2005) The economic burden of schizophrenia in the United States in 2002. Journal of Clinical Psychiatry 66(9): 1122-1129.

6. Bowers MB, Heninger GR, Gerbode F (1969) Cerebrospinal fluid 5-hydroxyindoleacetic acid and homovanillic acid in psychiatric patients. International journal of neuropharmacology 8(3): 255-262.

7. Levy MI, Davis BM, Mohs RC, Kendler KS, Mathe AA, et al. (1984) Apomorphine and schizophrenia: Treatment, CSF, and neuroendocrine responses. Archives of general psychiatry 41(5): 520-524.

8. Möller HJ (2003) Management of the negative symptoms of schizophrenia. CNS drugs 17(11): 793-823.

9. Leucht S, Corves C, Arbter D, Engel RR, Li C, et al. (2009) Secondgeneration versus first-generation antipsychotic drugs for schizophrenia: a meta-analysis. The Lancet 373(9657): 31-41.

10. Lane HY, Lin CH, Green MF, Hellemann G, Huang CC, et al. (2013) Addon treatment of benzoate for schizophrenia: a randomized, doubleblind, placebo-controlled trial of D-amino acid oxidase inhibitor. JAMA psychiatry 70(12): 1267-1275.

11. Kantrowitz JT, Woods SW, Petkova E, Cornblatt B, Corcoran CM, et al. (2015) D-serine for the treatment of negative symptoms in individuals at clinical high risk of schizophrenia: a pilot, double-blind, placebocontrolled, randomised parallel group mechanistic proof-of-concept trial. The Lancet Psychiatry 2(5): 403-412.

12. Correll CU, Rubio JM, Inczedy-Farkas G, Birnbaum ML, Kane JM, et al. (2017) Efficacy of 42 Pharmacologic Cotreatment Strategies Added to 
Antipsychotic Monotherapy in Schizophrenia: Systematic Overview and Quality Appraisal of the Meta-analytic Evidence. JAMA psychiatry $74(7): 675-684$

13. Tiihonen J, Mittendorfer-Rutz E, Majak M, Mehtälä J, Hoti F et al. (2017) Real-World Effectiveness of Antipsychotic Treatmnts in a Nationwide Cohort of 29823 Patients With Schizophrenia. JAMA psychiatry 74(7):686-693

14. McEvoy JP, Byerly M, Hamer RM, Dominik R, Swartz MS et al. (2014) Effectiveness of paliperidone palmitate vs haloperidol decanoate for maintenance treatment of schizophrenia: a randomized clinical trial. Jama 311(19): 1978-1987.

15. Mouchlianitis E, McCutcheon R, Howes (2016) Brain-imaging studies of treatment-resistant schizophrenia: a systematic review. The Lancet Psychiatry 3(5): 451-463.

16. Gage SH, Munafò MR (2015) Smoking as a causal risk factor for schizophrenia. The Lancet Psychiatry 2(9): 778-779.

17. Gurillo P, Jauhar S, Murray RM, Mac Cabe JH (2015) Does tobacco use cause psychosis? Systematic review and meta-analysis. The Lancet Psychiatry 2(8): 718-725.

18. Prochaska JJ (2010) Failure to treat tobacco use in mental health and addiction treatment settings: a form of harm reduction? Drug and alcohol dependence 110(3): 177-182.

19. Hong LE, Thaker GK, McMahon RP, Summerfelt A, RachBeisel J, et al. (2011) Effects of moderate-dose treatment with varenicline on neurobiological and cognitive biomarkers in smokers and nonsmokers with schizophrenia or schizoaffective disorder. Archives of general psychiatry 68(12): 1195-1206.

20. Dickinson D, Straub RE, Trampush JW, Gao Y, Feng N, et al. (2014) Differential effects of common variants in SCN2A on general cognitive ability, brain physiology, and messenger RNA expression in schizophrenia cases and control individuals. JAMA psychiatry 71(6): 647-656.

21. Keefe RS, Davis VG, Harvey PD, Atkins AS, Haig GM, et al. (2017) Placebo Response and Practice Effects in Schizophrenia Cognition Trials. JAMA psychiatry 74(8): 807-814.

22. Pekkala E, Merinder L (2002) Psychoeducation for schizophrenia. Cochrane Database Syst Rev 2(2).

23. Rathod S, Phiri P, Kingdon D (2010) Cognitive behavioral therapy for schizophrenia. Psychiatric Clinics of North America 33(3): 527-536.

24. Wykes T, Steel C, Everitt B, Tarrier N (2008) Cognitive behavior therapy for schizophrenia: effect sizes, clinical models, and methodological rigor. Schizophrenia bulletin 34(3): 523-537.

25. Sensky T, Turkington D, Kingdon D, Scott JL, Scott J, et al. (2000) A randomized controlled trial of cognitive-behavioral therapy for persistent symptoms in schizophrenia resistant to medication. Archives of general psychiatry 57(2): 165-172.

26. Pharoah F, Mari JJ, Rathbone J, Wong W (2010) Family intervention for schizophrenia. The Cochrane Library. Cochrane Database Syst Rev $8(12)$.

27. Pitschel-Walz G, Leucht S, Bäuml J, Kissling W, Engel RR (2015) The effect of family interventions on relapse and rehospitalization in schizophrenia: a meta-analysis. Schizophr Bull 27(1): 73-92.

28. Volkow ND (2009) Substance use disorders in schizophrenia-clinical implications of comorbidity. Schizophr Bull. 2009 May; 35(3): 469472 .

29. Carr V (1988) Patients' techniques for coping with schizophrenia: An exploratory study. Psychology and Psychotherapy: Theory, Research and Practice. Br J Med Psychol 61(4): 339-352.

30. Fiszdon JM, Kurtz MM, Choi J, Bell MD, Martino S (2015) Motivational interviewing to increase cognitive rehabilitation adherence in schizophrenia. Schizophrenia bulletin 42(2): 327-334.

31. Phutane VH, Thirthalli J, Kesavan M, Kumar NC, Gangadhar BN (2011) Why do we prescribe ECT to schizophrenia patients?. Indian journal of psychiatry 53(2): 149-151.

32. Kay SR, Fiszbein A, Opfer LA (1987) The positive and negative syndrome scale (PANSS) for schizophrenia. Schizophrenia bulletin 13(2): 261276.

33. Samara MT, Dold M, Gianatsi M, Nikolakopoulou A, Helfer B, et al. (2016) Efficacy, acceptability, and tolerability of antipsychotics in treatment-resistant schizophrenia: a network meta-analysis. JAMA psychiatry 73(3): 199-210.

\section{Your next submission with JuniperPublishers will reach you the below assets}

- Quality Editorial service

- Swift Peer Review

- Reprints availability

- E-prints Service

- Manuscript Podcast for convenient understanding

- Global attainment for your research

- Manuscript accessibility in different formats

( Pdf, E-pub, Full Text, Audio)

- Unceasing customer service

Track the below URL for one-step submission https://juniperpublishers.com/online-submission.php 